\title{
3D Printed Responsive Wood Interfaces: Shape-Changing Origami-Inspired Prototypes
}

\author{
Elena Vazquez \\ Pennsylvania State University | USA | emv10@psu.edu \\ Benay Gursoy \\ Pennsylvania State University | USA | bug61@psu.edu
}

\begin{abstract}
In this paper, we present a study for a 3D printed responsive wood interface in which we employed a scientific approach to assess the effects of various $3 \mathrm{D}$ printing parameters on shape-change. A full factorial design of experiments is conducted to determine the variables that maximize hygromorphic response. Analyzing the results of the experiments, we designed and fabricated origami-inspired prototypes, and tested their bimorph and gradient actuation. The contribution of this study to the growing body of literature on 3D printing responsive woodbased composites is the integration of gradient actuation and origami-inspired shape-changing strategies.
\end{abstract}

Keywords: Shape-change; Shape-changing materials; Material computation; 3D printed wood; Responsive architecture.

\section{INTRODUCTION}

What if our built environments adapt to changing environmental conditions by changing their shapes, colors, orientations, and material properties -- similar to living things in nature? What if the materials we use in our designs do not have static attributes but can change their shapes and properties? And what if we can design (with) this dynamic nature? Recent collaborative research efforts between materials science and design fields show that this is possible. Building envelopes that change their shapes in response to various stimuli, such as humidity, heat, light, etc. are among the outcomes of these collaborative efforts. Shape-change in these systems is embedded in the properties of the materials and does not require any additional electronic or mechanical control. This provides energy efficiency through better adaptability to environmental conditions -- just like how plants orient themselves to the sun, pinecones open and close in response to changing humidity levels to disperse their seeds, wet leaves curl as they dry.

This study seeks to develop design strategies for fabricating responsive wood interfaces. Previous studies have set out design strategies such as bilayering principles, kirigami geometries for shape amplification, and multimaterial approaches. This paper describes an experimental inquiry focusing on developing a variety of responsive hinges used to construct origami-inspired responsive units. After a preliminary study where we tested different possible geometries, we developed a full factorial design of experiments to check which variables can be used to maximize the bending response of responsive wood prototypes. After analyzing the results, we designed, fabricated, and tested a responsive installation with a gradient of hinge designs. This study combines an analytical methodology with an exploratory researchthrough-design approach in developing responsive wood interfaces. The paper contributes to the growing body of literature on $4 \mathrm{D}$ printing with wood-based composites, proposing a controlled bending response strategy.

\section{BACKGROUND}

\section{RESPONSIVE INTERFACES}

Recent advancements in materials science, synthetic biology, and engineering have provided new and exciting smart materials for architects and designers to design responsive interfaces. Smart materials have embedded sensors and actuators and can adjust their properties to changes in environmental conditions (Addington \& Schodek, 2005). The behaviors of smart materials, unlike complex mechanical systems, derive from interactions between engineered functional layers (Kretzer et al., 2012), making these materials suitable for designing lightweight and responsive systems. In recent years, designers have started to use smart materials to develop speculative installations that favor dynamism and interaction over static designs. An example is Shapeshift, an installation that explores the use of electroactive polymers at an architectural scale, proposing "organic" kinetic systems (Kretzer \& Rossi, 2012). Lumina, a responsive interface made with shape memory alloys and silicone skin, is another example of an organic user interface designed with building skin functionalities in mind (Khoo \& Salim, 2013). Franzke et al., (2016) developed hydroactive polymers, combining electroactive polymers and water for a futuristic "liquid-based architecture." These installations extend the current boundaries of smart materials from engineering to design, and present design strategies to incorporate these materials in future functional applications. Moreover, these installations are exploratory studies, and therefore, they open up new possibilities for sensory experiences.

\section{D PRINTING WOOD}

Architects and designers have used wood as a construction material for centuries due to its excellent mechanical properties and widespread availability. Traditionally, wood's hygromorphic qualities have not been desirable for designers. However, the emerging trend of designing dynamic and kinetic systems for the built environment from kinetic facades to self-assembled structures-, has 
resulted in the development of new materials and the reengineering of well-known materials such as wood (Vazquez, Randall, \& Duarte, 2019). New engineered materials such as electroactive polymers and shape memory alloys can be actuated on demand and are suitable for building shape-changing architectural skin systems (Kretzer \& Rossi, 2012; Khoo, Salim, \& Burry, 2011). Nonetheless, the reengineering of a well-known material such as wood presents a passive alternative to shape-change with low embodied energy, which can be fabricated with low cost and simple tools (Holstov, Farmer, \& Bridgens, 2017). This low-tech strategy follows biomimetic principles, taking inspiration from looking at shape-morphing elements in nature, such as pinecones and orchid seedpods.

One way of reengineering wood to take advantage of its hygromorphic properties relies on utilizing wood veneers and bilayer principles. In a bilayer configuration, two tightly bonded layers tend to bend when they present differential swelling. Therefore, by taking advantage of woods anisotropy (i.e., fiber orientation), one can design shapechanging wood structures that bend in response to humidity or water. Researchers have proposed several applications using bilayered wood veneers. Examples include self-forming building parts (Wood et al., 2018), an autonomous shading system (Vailati et al., 2018), and responsive building skins (Reichert et al., 2014). These studies demonstrate the potential of wood as a low-cost building element that can be used to construct innovative shape-morphing architectures.

Another approach is to utilize additive manufacturing or $3 \mathrm{D}$ printing to construct shape-changing wood composites in a process that is usually referred to as $4 \mathrm{D}$ printing. Research into 4D printing has rapidly grown since it was introduced in 2013 (Momeni et al., 2017). Additive manufacturing permits the fabrication of complex microstructures that can be designed to change shape. Self-assembled PLA structures that respond to temperature (An et al., 2018) and biomimetic hydrogel composites that swell in the presence of water (Gladman et al., 2016) are such examples. In the case of $4 \mathrm{D}$ printing wood composites, the extrusion process causes the alignment of wood fibers in the direction of printing due to shear-induced alignment (Le Duigou et al., 2016). The fibers' arrangement creates an anisotropic condition, which can be utilized to create layers with differential swelling that lead to a bending response in the presence of high relative humidity or water. A recent review by Le Duigou et al. (2020) identifies current trends in $3 D$ printing of natural fiber composites. It highlights the advantage of additive processes in fabricating complex shape-changing structures with innovative actuation mechanisms. Several studies have begun to examine how to $3 \mathrm{D}$ print responsive wood structures for architectural purposes. Correa et al. (2015) presented methods for developing hygroscopic wood transformations through differential printing methods, using wood filaments and polymers. A previous work by the authors formalized shape rules to compute the behavior of responsive wood elements (Vazquez et al., 2019). It proposed the use of kirigami geometries for amplifying the shapetransformation of 3D printed wood composites.
This study extends previous research on 3D printing responsive wood interfaces, combining an experimental design of experiments approach with the design and testing of prototypes. As an initial exploration, we designed and fabricated prototypes following three different strategies: linear elements with differential bending, origami-inspired elements with active hinges and non-active surfaces, and woven lattices (Figure 1). Among these preliminary prototypes, for this study, we have chosen to proceed with the origami-inspired ones to explore gradient actuation. In the second stage, we performed a design of experiments to test which variables significantly enhance the shapechanging response of the hinges. With the results obtained, we designed and fabricated a responsive installation comprising of nine modules in which we integrated hinges with different parameters to achieve a sequential folding response.

\section{METHODS}

In the first step, with the goal of developing a wood interface that can change its shape gradually in response to humidity, we designed and fabricated several prototypes based on our previous experiences on 3D printing woodbased composites and using a selection of strategies that we previously developed (Vazquez et al., 2019a; Vazquez et al., 2019b). These strategies include bilayering, controlling the layer order to design bending response, and coordinating tool-path orientation with the samples' overall geometric configurations. Three of the prototypes that we designed can be seen in Figure 1. We had an open-ended design approach in developing these prototypes and ran several iterations of $3 \mathrm{D}$ printing and actuating the prototypes to correct the discrepancies in tool-path generation. The second stage consisted of a "design of experiments". With this statistical method, we aimed to systematically determine the relationship between the variables of the process and the outputs. The design of experiments is a robust technique that has been primarily used in engineering fields to optimize products and processes (Ilzarbe et al., 2008). Recently, research in additive manufacturing adopted this methodology to understand the effects of process variables on the 3D printed outcome. For instance, Griffiths et al. (2016) optimized machine building parameters for energy use and waste using a design of experiments approach for using an extrusion-based manufacturing process. Similarly, Lanzotti et al. (2015) evaluated the effect of 3D printing settings (layer thickness, speed, and flow rate) on the geometry accuracy of built parts fabricated with a desktop printer. In this study, we utilize a design of experiments approach to identify the tool-path design variables that maximize the bending response. This approach enables to identify the statistically significant variables and to use those variables as design parameters for further exploratory studies. We propose that this combination of exploratory and analytical methods can help the designers understand and control the behavior of responsive materials, and design (with) these behaviors. 

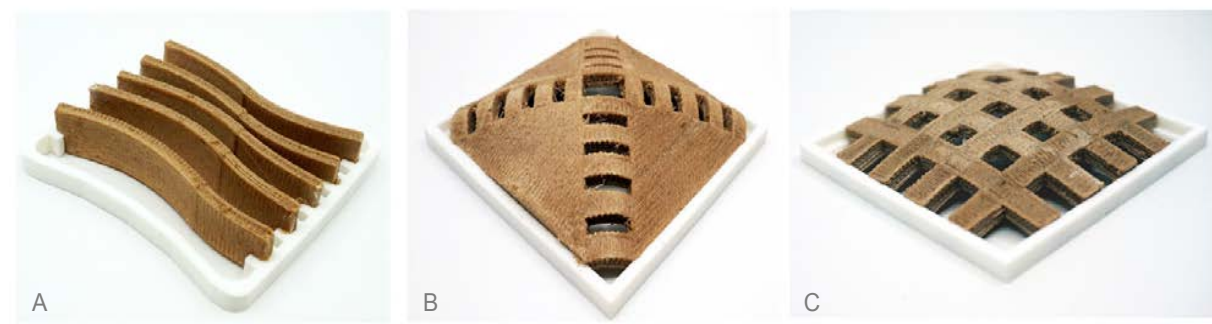

Figure 1: Three actuated prototypes for responsive wood interfaces: A. differential bending, B. origami-inspired, C. woven lattice.

\section{RESULTS}

\section{D PRINTED UNITS}

In the first stage of the study, we tested three different strategies for designing responsive interfaces. These strategies are linear elements with differential bending, origami-inspired elements with active hinges and nonactive surfaces, and woven lattices (Figure 1). With the differential bending prototypes, we aimed to create a gradient bending response by changing the order of layers. There are two types of layers in any bilayer configuration: Active Layers (AL) and Constraint Layers (CL). In the differential bending prototypes, active layers are perpendicular to the long side of the pieces. Therefore, the prototypes expand towards the longitudinal direction, and typically have more active layers than constraint layers. Constraint layers are parallel to the longitudinal direction of the prototypes. Their function, as their name suggests, is to constrain the expansion of the $\mathrm{AL}$, resulting in bending of the prototypes. These design strategies and a detailed explanation of $\mathrm{AL}$ and $\mathrm{CL}$ configurations in $3 \mathrm{D}$ printed responsive wood composites can be found in previously published works (Vazquez et al., 2018; Vazquez et al., 2019). Figure 1-A shows the resulting prototype of differential bending response, which shows the controlled bending in two directions conditioned by the AL and CL's layer order. We argue that this approach can be used to generate more complex compositions by employing simple $3 \mathrm{D}$ printed strips that bend to become single curved surfaces.

Origami-inspired prototypes combine specific geometrical configurations for bending surfaces (active) with nonbending (rigid) surfaces. Using origami geometries to achieve complex shape-morphing structures is not new in the area of $4 \mathrm{D}$ printing. The work by Zadpoor et al. (2017), for instance, demonstrated how to combine rigid and active parts in a folding configuration for extrusion-based additive manufacturing using PLA filaments. Similarly, our approach for origami-inspired responsive structures relies on identifying bending surfaces and designing these as hinges for the rigid parts. A key difference in using hygromorphic wood filament is the reversibility of the responsive behavior, making the system bimorph. Figure 1-B shows the resulting prototype of an actuated responsive pyramidlike structure. Rectangular hinges that connect the triangular surfaces in these prototypes start to bend when the humidity levels increase, which in return changes the overall form of the 3D printed prototype from a flat surface into a pyramidal form. The order of $A L$ and $C L$ of the hinges determines the bending direction.
The final exploration consists of weaving-inspired prototypes, of which one example is shown in Figure 1-C. A recent study by Grönquist et al. (2020) developed a similar approach to obtain double-curved surfaces by relying on the hygromorphic properties of wood. The study proposes the use of narrow wood-strip bilayers that selfshape into a grid shell structure. The prototype we present in Figure 1-C is based on the same approach of using shape-changing strips that are woven. However, our approach relies on additive manufacturing. Therefore, the strips are not discrete and do not necessitate additional connectors; They are already connected during the 3D printing process. Various weaving patterns can be explored using the same principle.

All three prototypes offer different potentials for a responsive interface. In this paper, we present our systematic explorations on the origami-inspired prototypes for developing a responsive interface. The possibility to discretize hinges offers more control over the design of the prototypes and the bending response. The combination of bending-active parts with rigid ones can also be employed in a multi-material $3 \mathrm{D}$ printing scheme by combining materials that are responsive with materials that are nonresponsive to humidity.

\section{DESIGN OF EXPERIMENTS}

The second stage of the study involves characterizing the behavior of the hinges of the origami-inspired prototypes to identify the tool-path design variables that maximize the bending response. In other words, to determine which variables are statistically significant in affecting the bending response of the hinges, so these can be used to design tailored bending in the next stages. With this goal in mind, we conducted an experiment with a $2^{2} 4$ multi-level full factorial design, with two replicates. We tested the significance of three factors or variables: Layer Height, Road Distance, and Active Layer/Constraint Layer Ratio, plus the interactions between these variables. Layer Height is the vertical distance between each layer of extrusion. Two settings were attributed to the Layer Height: the low value of $0.25 \mathrm{~mm}$ and a high value of $0.35 \mathrm{~mm}$, which are roughly within the standard values for extrusion-based processes. Road Distance is the horizontal distance between each linear extrusion. For Road Distance, the attributed low value was 0.7 , and the high value was 0.8 . We introduced 4 levels of Active Layer/Constraint Layer ratio, with the smallest value being 1 (same number of $A L$ and $\mathrm{CL}$ ) and the largest 4 (8 AL versus $2 \mathrm{CL}$ ). All $363 \mathrm{D}$ printed samples were placed in a humidity chamber for 12 hours. At the end of 12 hours, bending response was measured with a 3D printed jig that has multiple curvatures. 
A

$\begin{array}{lll}\text { Factors } & \text { Levels } & \text { Values } \\ \text { AC/CL Ratio } & 4 & 1,2,3,4 \\ \text { Layer Height } & 2 & 0.25,0.35 \\ \text { Road distance } & 2 & 0.7,0.8\end{array}$

C

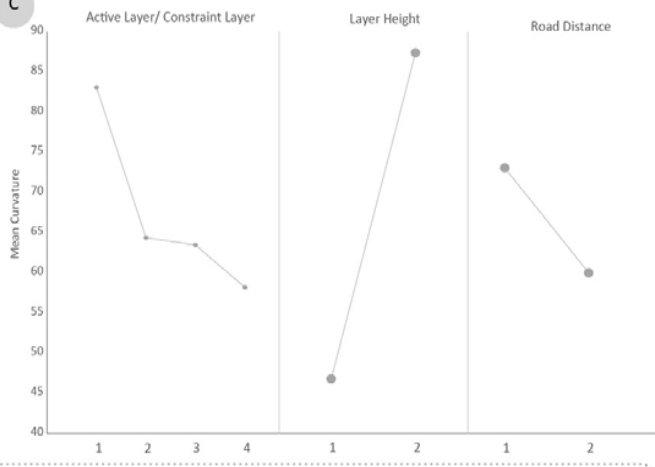

D

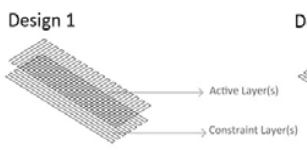

Design 2

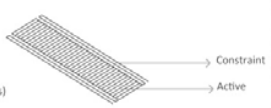

B

Layer Height* Road Distance

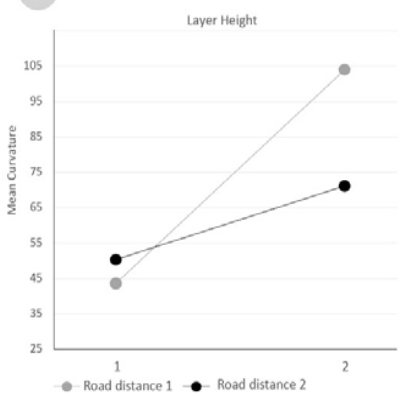

E

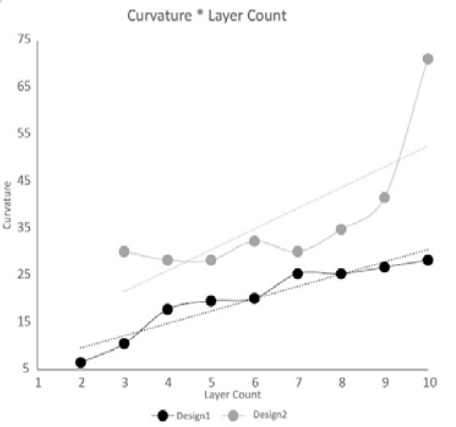

Figure 2: Graphs showing the results of the Design of Experiments

This benchmark specimen allowed for easy data collection. The tighter or smaller the curvature radius, the more bending that the samples present.

Figure 2 summarizes the results obtained after 3D printing, activating, and measuring all 36 prototypes. The data collected was analyzed with Minitab, a statistical analysis software. Figure 2-A summarizes the factors, number of levels utilized for the design of experiments, and the values assigned to each level. The results indicate that Layer Height and the combined effect of Layer Height and Road Distance are significant factors for the curvature response variable $(P<0.05)$. As shown in Figure $2-C$, curvature radius decreases (becomes tighter) with decreased Layer Height. The combined effect of Layer Height and Road Distance are also significant in predicting the bending response of the prototypes: a larger road distance $(0.8 \mathrm{~mm})$ results in a tighter curvature radius (Figure 2-B).

These results provided the tool-path design settings for the second round of experiments, in which we tested the effect of Total Layer Count on the bending response. Total Layer Count is considered a variable that has the potential for designing more complex responsive structures. Therefore, it was included in this second round, with all the other variables remaining constant in the settings that were found to maximize bending response. In this second round, we tested two different tool-path designs for the hinges (Design 1 and Design 2), as shown in Figure 2-D. Design 2 is based on research on $4 \mathrm{D}$ printing by Zadpoor et al. (2017). These two designs for the hinges have different approaches: in Design 1, actuation is due to bilayering, and in Design 2 actuation is constrained by strips on each side of the rectangle. The results for this second round of experiments are shown in Figure 2-E. Design 1 presented a tighter curvature radius than Design 2, and a clear trend between Total Layer Count measured curvature value. Lower values of Total Layer Count are associated with a tighter curvature radius for both Design 1 and Design 2. These results indicate that varying Total Layer Count in the prototypes can yield varied bending responses, and therefore can be used to achieve sequential bending in responsive hygromorphic structures. Figure $3-\mathrm{A}$ shows 9 actuated strips with Total Layer Counts decreasing from left to right from 10 to 2 layers.
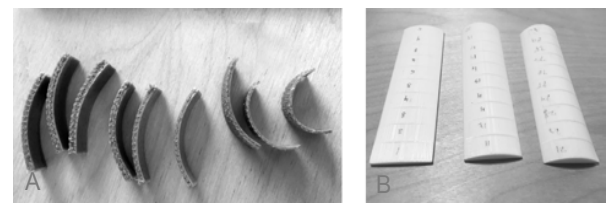

Figure 3: A. Strips with varied Total Layer Counts, B. 3D printed jigs to measure curvature of the strips.

\section{MODULAR RESPONSIVE SYSTEM}

Based on the results outlined above, we developed a modular system for a responsive interface using nine different variations of the origami-inspired prototype, as outlined in Figure 4-B. Variations in the prototypes are achieved by changing the tool-path configuration, specifically the Total Layer Count, of the hinges. Based on the previously articulated results of the experiments on 


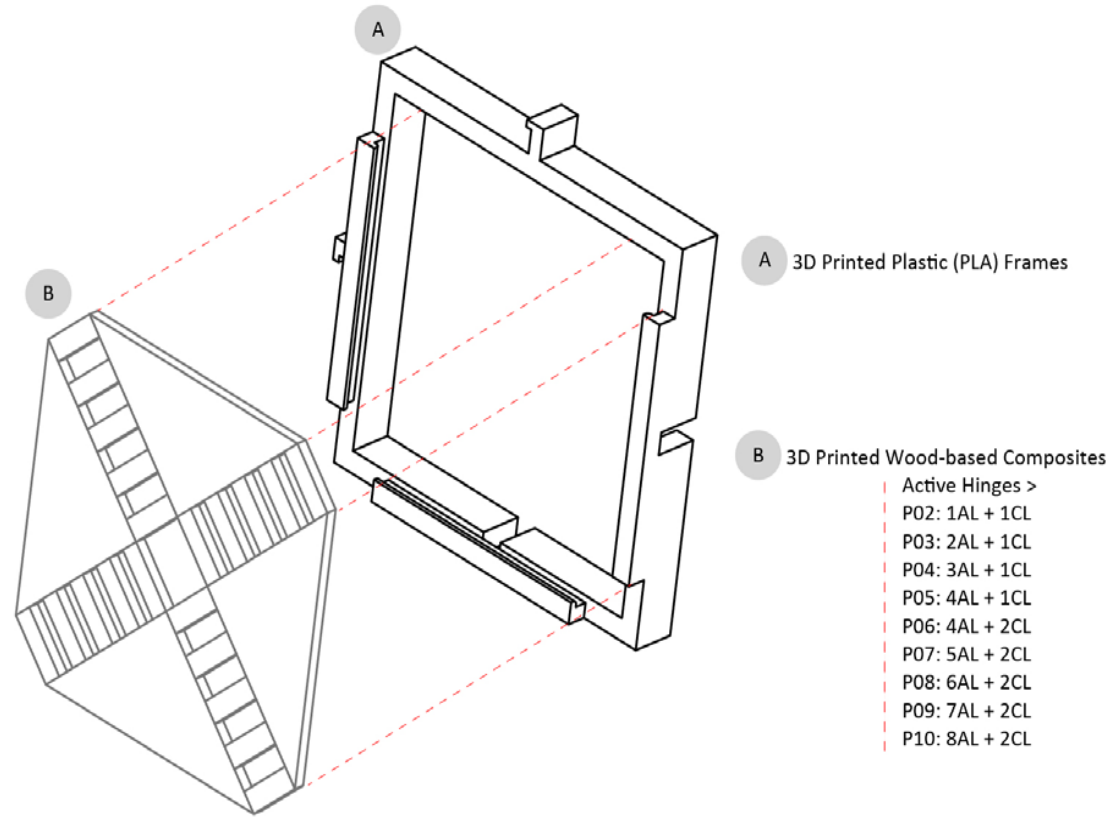

Figure 4 Modular system: A. 3D printed plastic frames, B. 3D printed wood-based composites

hinge designs, we hypothesized that hinges in the prototypes with higher Total Layer Count values would bend less or would take longer to bend compared to the hinges in the prototypes with lower Total Layer Count values. This would enable us to achieve two things: 1) a gradient bending/folding response of the prototypes at a given set time, and 2) sequential bending/folding of the prototypes over time.

To test this, we 3D printed two sets of all nine variations of the origami-inspired unit using the responsive wood-based filament. These wood-based parts are fixed on 3D printed plastic frameworks that serve to connect the units together while at the same time constraining the shape-change. Figure 4 outlines how the system works.

First, we placed the first set of units in the humidity chamber for 24 hours to check if the system works properly and to assess the resulting actuation. The humidity chamber is a transparent glass enclosure of 14 "x24" 117 " that has an opening of 14 "x 12 " at the bottom to locate the humidifier and dehumidifier interchangeably. The temperature of the humidity chamber is kept constant at 70 degrees Fahrenheit (21 degrees Celsius). To periodically document the shape-change in the units, we had to temporarily take the units out of the humidity chamber into the photo-studio.

The outcomes of this initial test can be seen in Figure 5. As expected, we documented a gradient bending/folding response in the units. The unit with the highest Total Layer Count value (P10) bent the least, and the unit with the lowest Total Layer Count value (P02) bent the most at the end of 24 hours. While the difference in actuation response between P10 and P02 was significant, the gradient transition between intermediary units $\mathrm{P} 03$ through $\mathrm{P} 09$ was negligible and difficult to perceive. This led us to run the second round of tests where we captured the shapechange hourly over a total of 6 hours. At the end of 6 hours, we replaced the humidifier in the chamber with a dehumidifier to explore the reversibility of the units' responsive behavior. Units remained in the chamber for another 6 hours, during which we retook hourly photos. Figure 6-A presents the photos taken every hour for 6 hours in the humidity chamber of the actuated units, and Figure 6-B presents the photos of the units taken every hour for 6 hours when the chamber is being dehumidified. Capturing the shape-change hourly enabled to better perceive the sequential bending/folding response of the units. However, while in the strip test (as can be seen in Figure 3-A), the gradient transition is more apparent, the difference between the origami-inspired prototypes is still negligible and hard to perceive. Even though the shape-change difference among the origami-inspired prototypes is negligible, each prototype significantly changes shape when the humidity levels change.

Among the nine prototypes, P04 with a Total Layer Count value of 4 (3AL and $1 \mathrm{CL}$ ) seemed to yield an "average" response. Therefore, we chose P04 to showcase the shape-change over time, both when the humidity levels increase and decrease. Figure $6-C$ illustrates the hourly shape-change in the individual unit P04 over 6 hours when the humidity level in the chamber is high $(99 \%)$. As can be seen, the shape-change of the prototypes is reversible: The 

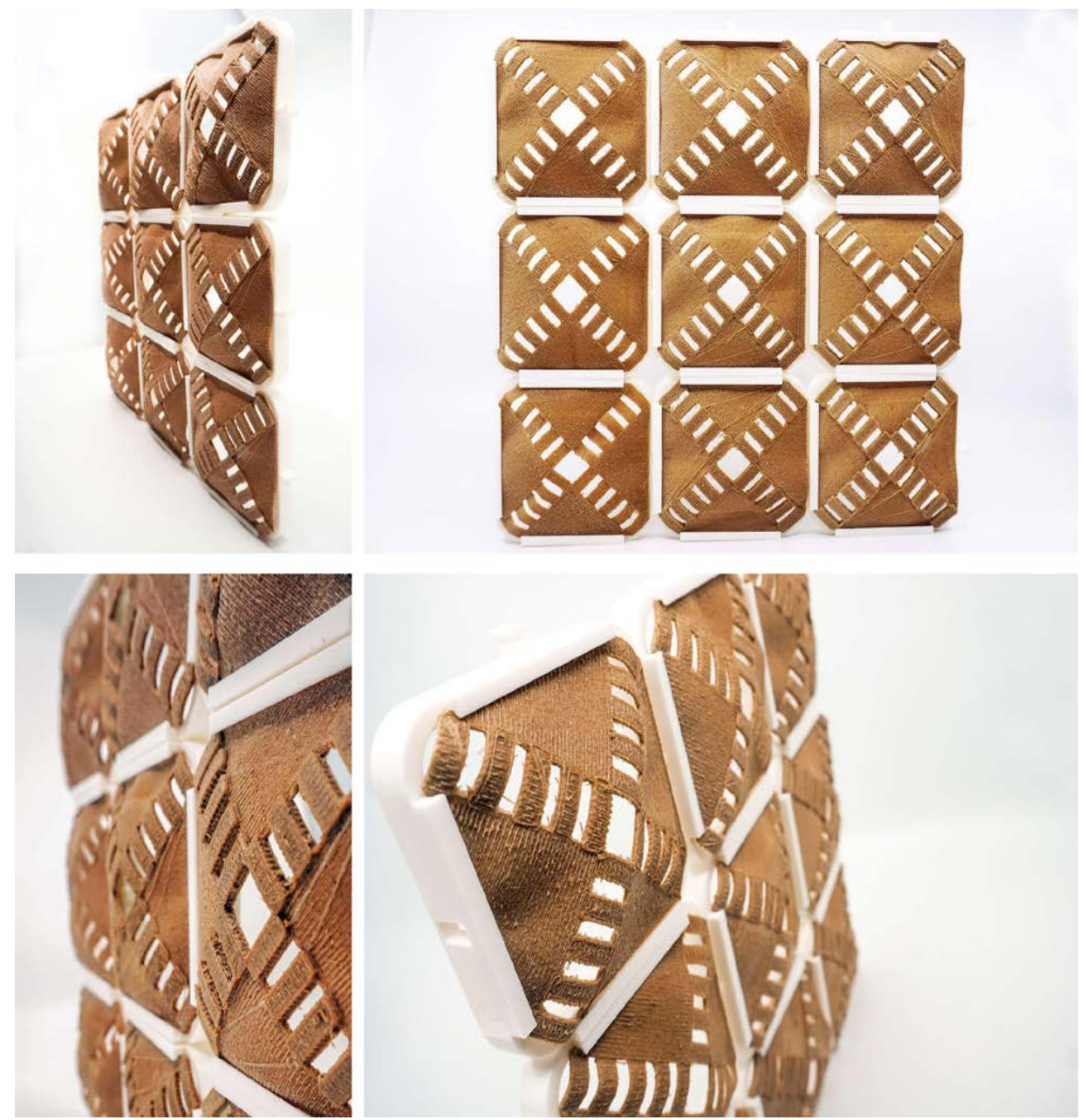

Figure 5 Prototypes actuated in the humidity chamber for 24 hours in $99 \%$ humidity levels

units return to their initial shape when the humidity level in the environment decreases from $99 \%$ to $35 \%$. This bimorph shape-change is promising and desirable in a responsive interface.

The structure can be expanded by adding more units in any direction. With more units of varied hinge configurations, it may be possible to perceive the gradient transition among the units better. It is also possible to change the $\mathrm{AL} / \mathrm{CL}$ order of the hinges to actuate the bending/folding shape change in the opposite direction.

\section{CONCLUSIONS}

In this paper, we presented a study for a responsive wood interface in which we 3D printed discrete units using a wood-based filament. The study contributes to the growing body of literature on 4D printing wood-based composites by 1) exemplifying how to implement a scientific approach to assess the effects of different printing parameters on shape-change, 2) integrating origami-inspired shape- change strategies, and 3) exploring sequential shapechange to create a gradient actuation.

Origami-inspired units presented in this paper use hinges to bend/fold the 3D printed flat surfaces. We adopted this hinge-logic from a study by Zadpoor et al. (2017) and updated it to use with wood-based filaments. Active hinges in this approach are used as substitutes for crease lines in origami. Bending/folding direction, similar to the valley and mountain folds in origami, can be controlled by adjusting the order of active layers $(A L)$ and constraint layers $(C L)$. It is possible to implement this logic to various, more complex origami patterns in which bending/folding occurs in multiple steps, and where there are no discrete units. One such exploration can be to integrate the hinge-logic to curvedcrease folding patterns. As we have shown in this paper, changing the tool-path configuration of hinges enables to control the actuation response. This way, it is possible to create responsive origami-inspired systems with sequential bending/folding. 

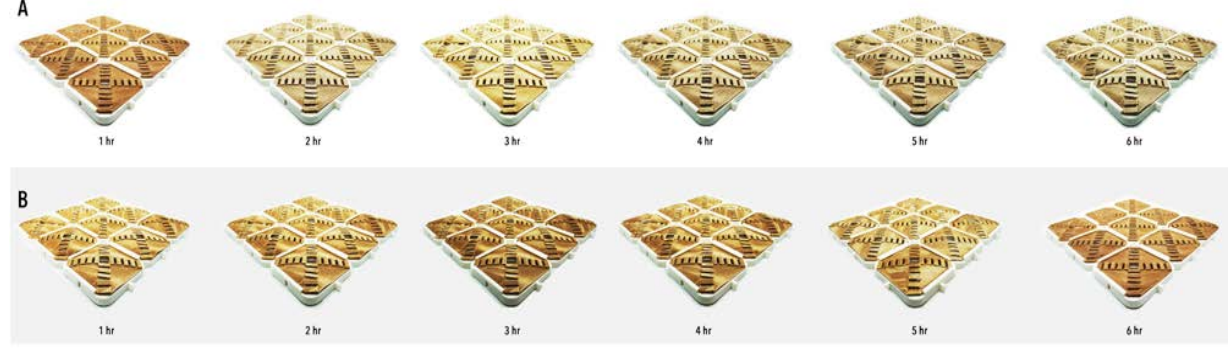

C
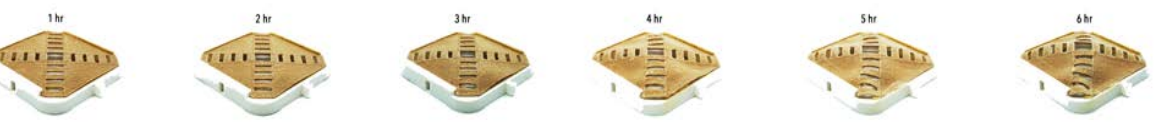

Figure 6 Units captured hourly: A. 99\% relative humidity, B. 35\% relative humidity, C. Prototype P04 in 99\% relative humidity

Shape-change in responsive systems is typically achieved by integrating complex mechanical structures or custommade expensive smart materials. Creating a shapechanging responsive interface by $3 \mathrm{D}$ printing using woodbased filaments is, on the other hand, accessible and affordable. The prototypes and the modular assembly system presented in this paper are fabricated using widely available desktop 3D printers, and shape-change is actuated through changes in the relative humidity levels. Therefore, developing an open-source platform for designing and fabricating hygroscopic shape-changing systems by $3 \mathrm{D}$ printing wood-based composites is possible. As previous studies on 4D printing show, these kinds of systems with responsive wood can be used for multiple applications, ranging from responsive architectural screens to self-assembling furniture. The downside is the limited printing area of standard desktop 3D printers, which can be overcome by developing custom machines if larger print areas are necessary.

We have previously developed a shape grammar to formalize shape-change in 3D printed wood-based kirigami geometries (Vazquez et al., 2019b). A future study is to develop a similar "motion grammar" to formalize the bending mechanism of the origami-inspired prototypes that we presented. This could be useful to generalize the rules and variables for $3 \mathrm{D}$ printing origami-inspired composites and help designers to understand and predict the shapechanging response that they can obtain based on the 3D printing parameters and the auxiliary parameters that are related to environmental conditions.

Finally, it is essential to acknowledge that as designers, our approach to understanding the shape-changing behavior of materials is different than that of materials scientists. We are not after modeling the behavior of responsive 3D printed wood with scientific precision. Rather, we are exploring ways to use this behavior in a controlled manner and design (with) it. Therefore, bending behavior is not the ultimate goal, but rather it is the means to an end in the design process. Design processes are creative processes that rely on uncertainties. In the case of shape-changing systems, the uncertainty of material behavior over time is what enriches this process.

\section{ACKNOWLEDGEMENTS}

This research is supported by The $\mathrm{H}$. Campbell an Eleanor R. Stuckeman Fund for Collaborative Design Research at The Pennsylvania State University.

\section{REFERENCES}

Addington, M., \& Schodek, D. (2005). Smart materials and technologies. $A+U$ Architecture and Urbanism, (412).

An, B., Tao, Y., Gu, J., Cheng, T., Chen, X. "Anthony," Zhang, X., ... Yao, L. (2018). Thermorph: Democratizing 4D Printing of Self-Folding Materials and Interfaces. Proceedings of the 2018 $\mathrm{CHI}$ Conference on Human Factors in Computing Systems,260:1--260:12.

https://doi.org/10.1145/3173574.3173834

Correa, D., Papadopoulou, A., Guberan, C., Jhaveri, N., Reichert, S., Menges, A., , S. (2015). 3D-Printed Wood: Programming Hygroscopic Material Transformations. $3 D$ Printing and Additive Manufacturing, 2(3), 106-116. https://doi.org/10.1089/3dp.2015.0022

Franzke, L., Rossi, D., \& Franinović, P. K. (2016). Fluid Morphologies. Hydroactive Polymers for Responsive Architecture. Acadia, (October), 478-487.

Gladman, A. S., Matsumoto, E. A., Nuzzo, R. G., Mahadevan, L., \& Lewis, J. A. (2016). Biomimetic 4D printing. Nature Materials, 15(April). https://doi.org/10.1038/NMAT4544

Griffiths, C. A., Howarth, J., De Almeida-Rowbotham, G., Rees, A., \& Kerton, R. (2016). A design of experiments approach for the optimisation of energy and waste during the production of parts manufactured by 3D printing. Journal of Cleaner Production, 139, 74-85.

Grönquist, P., Panchadcharam, P., Wood, D., Menges, A., Rüggeberg, M., \& Wittel, F. K. (2020). Computational analysis of hygromorphic self-shaping wood gridshell structures. Royal Society Open Science, 7(192210).

Holstov, A., Farmer, G., \& Bridgens, B. (2017). Sustainable materialisation of responsive architecture. Sustainability (Switzerland), 9(3). https://doi.org/10.3390/su9030435

Ilzarbe, L., Alvarez, M. J., Viles, E., \& Tranco, M. (2008). Practica applications of design of experiments in the field of engineering: a bibliographical review. Quality and Reliability Engineering International, 24(4), 417-428.

Khoo, C. K., Salim, F., \& Burry, J. (2011). Designing Architectural Morphing Skins with Elastic Modular Systems. International Journal of Architectural Computing, 09(04), 397-419. https://doi.org/10.1260/1478-0771.9.4.397 
Khoo, C. K., \& Salim, F. D. (2013). Lumina : A Soft Kinetic Material for Morphing Architectural Skins and Organic User Interfaces. In Proceedings of the 2013 ACM international joint conference on Pervasive and ubiquitous computing (pp. 53-62). New York, N.Y.: Association for Computing Machinery. https://doi.org/10.1145/2493432.2494263

Kretzer, M., Minuto, A., \& Nijholt, A. (2012). Smart material interfaces: a material step to the future. Proceedings of the $1 \mathrm{st}$ Workshop on Smart Material Interfaces: A Material Step to the Future - SMI'12, 615-616. https://doi.org/10.1145/2459056.2459057

Kretzer, M., \& Rossi, D. (2012). ShapeShift. Leonardo, 45(5), 480 481. Retrieved from https://www.muse.jhu.edu/article/484764.

Lanzotti, A., Martorelli, M., \& Staiano, G. (2015). Understanding process parameter effects of reprap open-source threedimensional printers through a design of experiments approach. Journal of Manufacturing Science and Engineering, 137(1).

Le Duigou, A., Castro, M., Bevan, R., \& Martin, N. (2016). 3D printing of wood fibre biocomposites: From mechanical to actuation functionality. Materials and Design, 96, 106-114. https://doi.org/10.1016/j.matdes.2016.02.018

Le Duigou, Antoine, Correa, D., Ueda, M., Matsuzaki, R., \& Castro, M. (2020). A review of $3 D$ and 4D printing of natural fibre biocomposites. Materials \& Design, 194, 108911. https://doi.org/10.1016/j.matdes.2020.108911

Momeni, F., Liu, X., \& Ni, J. (2017). A review of 4D printing. A Review of 4D Printing, 122, 42-79.

Reichert, S., Menges, A., \& Correa, D. (2014). Meteorosensitive architecture: Biomimetic building skins based on materially embedded and hygroscopically enabled responsiveness. CAD Computer Aided Design, 60, 50-69. https://doi.org/10.1016/j.cad.2014.02.010
Tibbits, S., McKnelly, C., Olguin, C., Dikovsky, D., \& Hirsch, S (2014). 4d Printing and Universal Transformation. ACADIA 14 Design Agency: Proceedings of the 34th Annual Conference of the Association for Computer Aided Design in Architecture, 539-548.

Vailati, C., Bachtiar, E., Hass, P., Burgert, I., \& Rüggeberg, M. (2018). An autonomous shading system based on coupled wood bilayer elements. Energy and Buildings, 158, 10131022. https://doi.org/10.1016/j.enbuild.2017.10.042

Van Manen, T., Janbaz, S., \& Zadpoor, A. A. (2017). Programming 2D/3D shape-shifting with hobbyist $3 D$ printers. Materials Horizons, 4(6), 1064-1069. https://doi.org/10.1039/c7mh00269f

Vazquez, E., Randall, C., \& Duarte, J. P. (2019). Shape-changing architectural skins: a review on materials, design and fabrication strategies and performance analysis. Journal of Facade Design and Engineering, 7(2), 91-102.

Vazquez, E., Gursoy, B., \& Duarte, J. (2019a). Designing for shape change - A case study on 3D printing composite materials for responsive architectures. In: Intelligent \& Informed Proceedings of the 24th CAADRIA Conference - Volume 2 (eds M Haeusler, MA Schnabel, and T Fukuda), 15-18 April 2019, pp. 391-400. Wellington: Victoria University of Wellington.

Vazquez, E., Gursoy, B., \& Duarte, J. (2019b). Formalizing ShapeChange in Design: 3D Printed Shapes and Hydro-Responsive Material Transformations. International Journal of Architectural Computing, Volume: 18 issue: 1, page(s): 1-17. DOI: $10.1177 / 1478077119895216$.

Wood, D., Vailati, C., Menges, A., \& Rüggeberg, M. (2018). Hygroscopically actuated wood elements for weather responsive and self-forming building parts - Facilitating upscaling and complex shape changes. Construction and Building Materials, 165, 782-791. https://doi.org/10.1016/j.conbuildmat.2017.12.134 